\title{
Ultraviolet, Circular Dichroism, Fluorescence, and Circularly Polarized Luminescence Spectra of Regioregular Poly-[3-((S)-2-Methylbutyl)-Thiophene] in Solution
}

\author{
ETTORE CASTIGLIONI, ${ }^{1,2}$ SERGIO ABBATE, ${ }^{1,3 *}$ FRANCE LEBON, ${ }^{1,3}$ AND GIOVANNA LONGHI ${ }^{1,3}$ \\ ${ }^{1}$ Dipartimento di Scienze Biomediche e Biotecnologie, Università di Brescia, Brescia, Italy \\ ${ }^{2}$ Jasco Europe, Cremella (LC), Italy \\ ${ }^{3}$ Consorzio Nazionale Interuniversitario per le Scienze Fisiche della Materia, Rome, Italy
}

\begin{abstract}
Ultraviolet (UV), Circular Dichroism (CD), Fluorescence, and Circularly Polarized Luminescence (CPL) spectra were recorded for mixed chloroform/methanol and chloroform/ butanol solutions of regioregular poly [3-((S)-2-methylbutyl)-thiophene]. An evaluation of the chiral and nonchiral components in solution is proposed on the basis of the UV and CD spectra. The fluorescence and CPL spectra are observed in a wavelength range where some UV and CD activity still appears. For this reason, the observed CPL features are strongly influenced by CD. A general procedure for evaluating true CPL spectra is proposed, when absorption and emission coexist. Chirality 00:000-000, 2012. ( 2012 Wiley Periodicals, Inc.
\end{abstract}

KEY WORDS: circular dichroism (CD); circularly polarized luminescence (CPL); polyalkylthiophenes; $g$ factor

\section{INTRODUCTION}

Chiral polyalkylthiophenes (PAT) are a class of largely studied polymers (see Scheme 1), which, because of the presence of the conjugated backbone, are conducting organic molecular systems and, because of the chiral substituent X, may provide additional properties related to symmetry breaking, such as large second-order susceptibility constants. To this scope, $X$ has to be substituted regioregularly along the polythiophene chain, as first demonstrated by LangeveldVoss $e t a l^{1,2}$ However, even though there is a general feeling and a large consensus on how optical activity is generated, both in absorption and emission, we have to admit that the understanding of the chiral phenomena in these systems is only qualitative. Lately though, a number of theoretical papers have reported new or updated models for understanding the Circular Dichroism (CD) and Circularly Polarized Luminescence (CPL) data of PAT. ${ }^{3,4}$ In the past, in our group, we were able to synthesize and characterize a PAT polymer with a very short X substituent group ((S)-2-methylbutyl moiety) (PMBT) and a high degree of regioregularity: ${ }^{5-8}$ chemicophysical methods for the structural characterization included, besides other spectroscopic methods, regular electronic $\mathrm{CD}^{5-7}$ as well as Infrared or Vibrational CD. ${ }^{8}$ The special properties and cleanliness of that PAT allowed its full X-ray characterization. ${ }^{9}$ Besides, we were able to verify ${ }^{7}$ what had been shown previously for other PAT systems, ${ }^{2}$ namely, in good solvents, such as $\mathrm{CHCl}_{3}$, $\mathrm{PMBT}$ exhibits no $\mathrm{CD}$ spectra, whereas in good solvent/bad solvent mixtures (e.g., $\mathrm{CHCl}_{3}$ / $\mathrm{CH}_{3} \mathrm{OH}$ ), PMBT exhibits remarkable $\mathrm{CD}$. In the latter case, a chiral phase appears, which manifests itself in special circumstances and in the solid state, that is, with adequate sample preparation. ${ }^{7}$ Because we recently assembled a rather versatile apparatus for measuring CPL, ${ }^{10}$ and because of the increased use of the latter technique, ${ }^{11-14}$ we wish to complete the characterization of our PAT system with the aim of comparing with the CPL data of the existing literature ${ }^{1,15}$ and to provide some new material for the theoreticians to ponder.

(C) 2012 Wiley Periodicals, Inc

\section{EXPERIMENTAL}

The PMBT sample employed here is the same as we had synthesized and studied 10 years ago. ${ }^{5-8}$ Electronic $\mathrm{CD}$ spectra were recorded in chloroform/methanol mixed solvents as in Ref. 5 on a new apparatus, Jasco 815SE; in addition, chloroform/(S)-(+)-2-butanol mixtures were investigated (even $(R)-(-)$-2-butanol was employed, but no appreciable differences were noticed between the spectra taken in the two enantiomeric solvents). The experimental conditions were: $2 \mathrm{~mm}$ cuvette, $5.3 \times 10^{-4} \mathrm{M}$ concentration, $100 \mathrm{~nm} / \mathrm{min}$ scanning speed, two scans per measurement, and $2 \mathrm{sec}$ integration time. Differently from our previous experiments, absorption spectra have been collected simultaneously to $\mathrm{CD}$ to minimize anomalies induced by separate measurements on instruments with different sampling geometries. The very same solutions were then used for the CPL experiments in the following conditions: $90^{\circ}$ geometry, $2 \mathrm{~mm}$ fluorescence semimicro cuvette $(2 \mathrm{~mm}$ path in the excitation side, $10 \mathrm{~mm}$ path in the emission direction), $75 \mathrm{~nm} / \mathrm{min}$ scanning speed, five scans per measurement, and $2 \mathrm{sec}$ time constant. The CPL apparatus was the one we described in Ref. 10, which was since updated, by interfacing it with a Jasco 8200 Fluorimeter. The latter instrument allows one to quickly obtain luminescence spectra, permitting an overview of the emission properties and fast decision on how to excite the system and may also act as a continuously variable source of radiation for the CPL home-built module, to which light is brought through a water-filled optical fiber. The necessary electronics to interface the analog outputs of our spectrometer and the buffer to control the scanning motor has been built around a Data Translation card, with the addition of an opportune software to acquire simultaneously Fluorescence (from DC signals) and CPL (from AC signals) spectra and perform spectra accumulations. ${ }^{16}$

Because it is well known from the literature ${ }^{17-19}$ that CPL spectra are prone to artifacts caused by linear polarization components, before proceeding to comment our results, we wish to report on a systematic investigation to detect the presence of linearly polarized components in

\footnotetext{
${ }^{*}$ Correspondence to: Sergio Abbate, Dipartimento di Scienze Biomediche e Biotecnologie, Università di Brescia, Viale Europa 11, 25123 Brescia, Italy. E-mail: abbate@med.unibs.it

Received for publication 30 November 2011; Accepted 18 January 2012

DOI: $10.1002 /$ chir.22023

Published online in Wiley Online Library

(wileyonlinelibrary.com).
} 


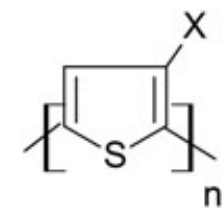

$$
\mathrm{X}=\mathrm{CH}_{2}-\mathrm{C} * \mathrm{H}\left(\mathrm{CH}_{3}\right)-\mathrm{CH}_{2}-\mathrm{CH}_{3}
$$

Scheme 1. Structure of the poly[3-((S)-2-methylbutyl)-thiophene] (PMBT).

the PMBT emission spectra recorded with the same operating conditions adopted throughout this work. The test conditions were (1) the sample is irradiated at $90^{\circ}$ by the light generated by the fluorimeter, through an optical fiber; the light may be polarized by placing a linear polarizer before the sample. (2) We normally use a $50-\mathrm{kHz}$ lock-in amplifier (same frequency as the photoelastic modulator) to detect CPL, but we may also use a $100-\mathrm{kHz}$ lock-in amplifier, to detect Linearly Polarized Luminescence features. The possible linear polarization effects and the influence onto CPL spectra are illustrated in Figure 1; we examined the case of PMBT 100\% chloroform solutions, which are known to be optically inactive (vide infra and see also Refs. 2 and 5-8). The shape of CPL spectra is the same as the fluorescence spectra (they are just less intense) in two cases: either when the polarizer is absent or when it is oriented in the vertical position; on the contrary, CPL signal is null, as it should, when the polarizer is in the horizontal position. Consequently, all CPL spectra have then been collected inserting a horizontal polarizer to eliminate linear polarization components from the CPL data. Furthermore, the absence of linear polarization effects has been confirmed (data not shown) by collecting a zero output signal, as measured by $100-\mathrm{KHz}$ lock-in amplifier, in correspondence of all CPL data presented here. Similarly, vanishingly small linear dichroism data have been recorded simultaneously to the $\mathrm{CD}$ absorption spectra.
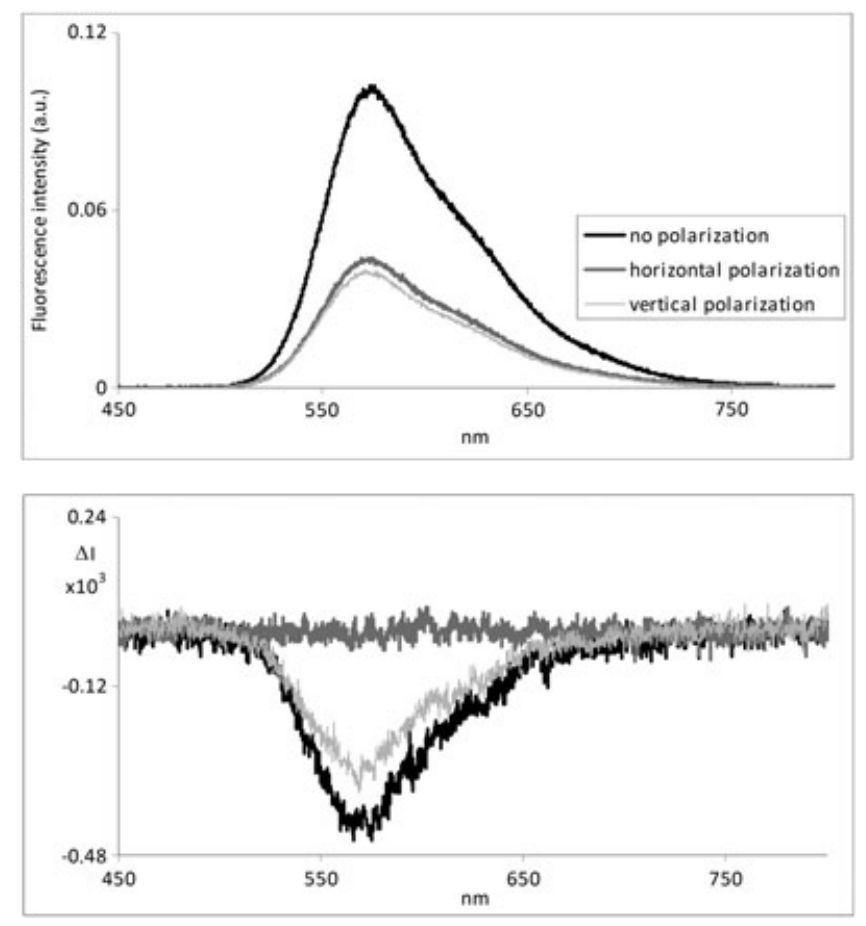

Fig. 1. Emission (top) and circularly polarized luminescence spectra (excitation at $400 \mathrm{~nm}$ ) of chloroform solution of poly[3-((S)-2-methylbutyl)thiophene] in a 2 -mm cell for exciting radiation with no polarization (black bold trace), with horizontal polarization (gray bold trace) and with vertical polarization (gray trace).

Chirality DOI: 10.1002/chir

\section{RESULTS AND DISCUSSION}

In Figures 2 and 3, we report the spectra for UV absorption (top left), CD (middle left), $g_{\text {abs }}$ (bottom left), fluorescence (top right), measured CPL (middle right), and $g_{\text {lum }}$ (bottom right) of two kinds of mixed solutions of PMBT, namely the $\mathrm{CHCl}_{3} /(\mathrm{S})$-(+)-2-butanol and the $\mathrm{CHCl}_{3} / \mathrm{CH}_{3} \mathrm{OH}$ solutions, respectively, at varying percentage of the two solvents (in the figures, we provide the volume per cent content of $\mathrm{CHCl}_{3}$ ). The $g_{\text {abs }}$ and $g_{\text {lum }}$ factors are the ratios of $\mathrm{CD}$ and UV spectra and of CPL and Fluorescence spectra, respectively. ${ }^{10,17,20}$ The latter two spectra were obtained by exciting the sample at $470 \mathrm{~nm}$ for the mixed chloroform-butanol solutions and at $493 \mathrm{~nm}$ for the mixed chloroform-methanol solutions because the two wavelengths approximately are in correspondence with isosbestic points in the two respective UV spectra; this permits to have the same absorption for all solutions and thus to study CPL and fluorescence phenomena for all cases in Figures 2 and 3 on common grounds. We report $g_{\text {lum }}$, because CPL data are often presented in terms of dissymmetry factor, which is an absolute quantity; however, as discussed in the succeeding text, the data presented in these figures are the "apparent" CPL and $g_{\text {lum }}$ and cannot be compared with $g_{\text {abs }}$ without due corrections. In any case, referring to the dissymmetry factor, we checked our instrument with a standard used in the literature, Europium tris[3-(trifluoromethylhydroxymethylene)-(+)-camphorate] in dimethylsulfoxide solution, obtaining a value $g_{\text {lum }} \sim-0.7$ for the ${ }^{5} D_{0} \rightarrow{ }^{7} F_{1}$ transition. ${ }^{21,22}$

The interpretation of the observed features is not immediate; we are in presence of a heterogeneous system, with two clearly defined components, as appears from the well-defined isosbestic point in the UV spectra in Figure 2, and, to a slightly lesser degree, in the UV spectra of Figure 3. One of the two components is optically inactive, and its UV spectrum is the one in 100\% chloroform (indeed its CD spectrum is flat). We can evaluate the content of this optically inactive component in the various mixed solvents by assuming that its amount progressively decreases with addition of "bad" solvent (either butanol or methanol) until it disappears in the last investigated solution (beyond which the polymer precipitates). The last situation is met when we are in presence of $60: 40 \mathrm{CHCl}_{3} /$ butanol v/v ratio; here, we have $0 \%$ optically inactive PMBT. The content of optically inactive phase at $70: 30(\mathrm{v} / \mathrm{v})$ is then $23 \%$, at $80: 20(\mathrm{v} / \mathrm{v})$ is $76 \%$, and of course, at $100: 0(\mathrm{v} / \mathrm{v})$ is $100 \%$. In the case of $\mathrm{CHCl}_{3} /$ methanol mixed solutions, the phenomena show up in a more restricted interval and are such that at $87: 13(\mathrm{v} / \mathrm{v}) \mathrm{CHCl}_{3} /$ methanol, the optically inactive component is $0 \%$; then, at $88: 12(\mathrm{v} / \mathrm{v})$, it is $16 \%$; at $90: 10(\mathrm{v} / \mathrm{v})$, it is $37 \%$; at $93: 7(\mathrm{v} / \mathrm{v})$, it is $90 \%$; finally at $100: 0(\mathrm{v} / \mathrm{v})$, it is $100 \%$. If we subtract the thus weighted absorption of the nonchiral component from the UV spectra at the various concentrations, we can obtain the UV spectra of just the optically active polymeric aggregate. The results are given in the insets $\mathrm{A}$ of the Figures 2 and 3; one may notice that the UV spectra of the optically active PMBT polymers are increasing much in the same way as the CD spectra of the same figures. Moreover, and most importantly, the recalculated (insets B) $g_{\text {abs }}$ spectra are constant, which means that, independently of its content, the optically active component is the same and thus possesses the same "intrinsic" CD spectrum.

The major difficulty in the interpretation of emission data is the fact that recorded fluorescence and CPL spectra are in a 

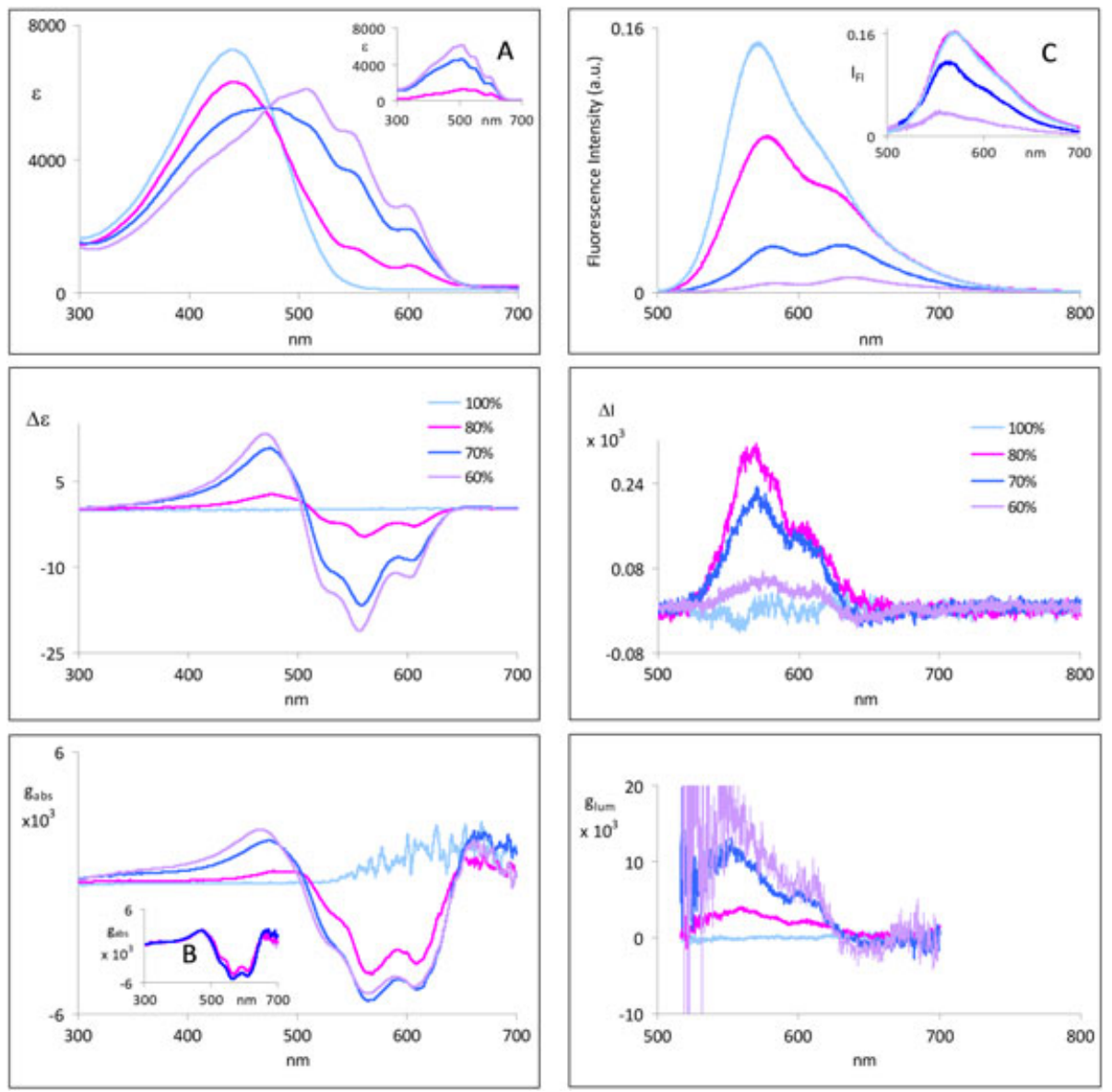

Fig. 2. (left panels) Ultraviolet (top), electronic circular dichroism (middle), and $g_{\text {abs }}$ (bottom) spectra of poly[3-((S)-2-methylbutyl)-thiophene] (PMBT) in different $\mathrm{CHCl}_{3}$ /butanol mixtures. Inset A: ultraviolet spectra of the chiral component. Inset B: $\mathrm{g}_{\text {abs }}$ spectra of the chiral component. (right panels) Emission (top), circularly polarized luminescence (CPL) (middle), and $g_{\text {lum }}$ (bottom) spectra of PMBT in different $\mathrm{CHCl}_{3}$ /butanol mixtures with a 470-nm excitation. CPL and $g_{\text {lum }}$ are not corrected; for this reason, $g_{\text {lum }}$ cannot be compared with $g_{\text {abs. }}$. Inset C: corrected luminescence spectra, accounting for absorption of emitted light.

wavelength range where there is still strong absorption from the sample. This inevitably alters intensity and shape of both fluorescence and CPL bands. In the present case, we have used a $2 \times 10$-mm cell, and the average emission path is therefore about $5 \mathrm{~mm}$; on the other hand, the size of the excitation fiber is such that it does not allow the use of smaller cells. We evaluated a correction for fluorescence spectra by first calculating the transmittance spectra corresponding to the UV-absorption spectra of Figures 2 and 3 with the assumption of $5 \mathrm{~mm}$ pathlength and by then dividing total emission spectra by the obtained results for transmittance spectra. No correction has been applied for the absorption of the excitation beam because the excitation wavelength was chosen at the isosbestic point. The corrected fluorescence spectra are given in insets $\mathrm{C}$ of the two Figures; the intensity of emission bands as function of the relative concentration of the two solvents in the mixture has an opposite trend with respect to corresponding absorption spectra.

We are aware that also CPL spectra are affected by these same factors and that additionally, a "chiral" correction, to be evaluated from the CD curve, should be applied, and that will be described next. However, before then, we wish to report that we excited the systems both at longer and shorter wavelength, but we did not get qualitatively different spectra for exciting radiation with wavelength in the 350-550-nm range (outside that interval we were not able to obtain any CPL spectra). In particular, we did not record any negative feature. To understand to what extent, the CPL-observed spectra are influenced by underlying CD phenomena affecting the emitted radiation; we tried to estimate the level of the CD signal coming from the fluorescence emitted light, which is absorbed by our samples. In our apparatus, this procedure is made possible by the fact that the CPL scale is calibrated in millideg of ellipticity for unitary value of fluorescence.$^{10}$ So, we divided the CPL raw data changed in sign by the fluorescence data; the change in sign is required by the fact that we are now considering absorption rather than emission phenomena. The results are then compared with the raw CD data with millideg scale obtained with the J-815SE apparatus on the same solutions in 2-mm pathlength cuvettes, multiplied by 2.5 to compensate the different pathlength. The set of mathematical operations performed on the CPL data parallels the running of a $\mathrm{CD}$ spectrum, which is obtained by dividing an AC signal (corresponding to -CPL here) by a DC signal (corresponding to Fluorescence here). ${ }^{10,23}$ In Figure 4, we superimpose the $\mathrm{CD}$ spectra obtained in this way to the "regular" CD spectra presented in Figures 2 and 3 for the chloroform/butanol and chloroform/methanol cases, obtained on the standard Jasco J815SE machine. We may see that the two differently obtained CD spectra are quite similar, except for the obvious fact that the so-called CPL-detected $\mathrm{CD}$ spectra are much noisier than regular $\mathrm{CD}$ spectra because the light intensity in fluorescence (DC signal) is much smaller than the one used for transmission experiments. This 
CASTIGLIONI ET AL.
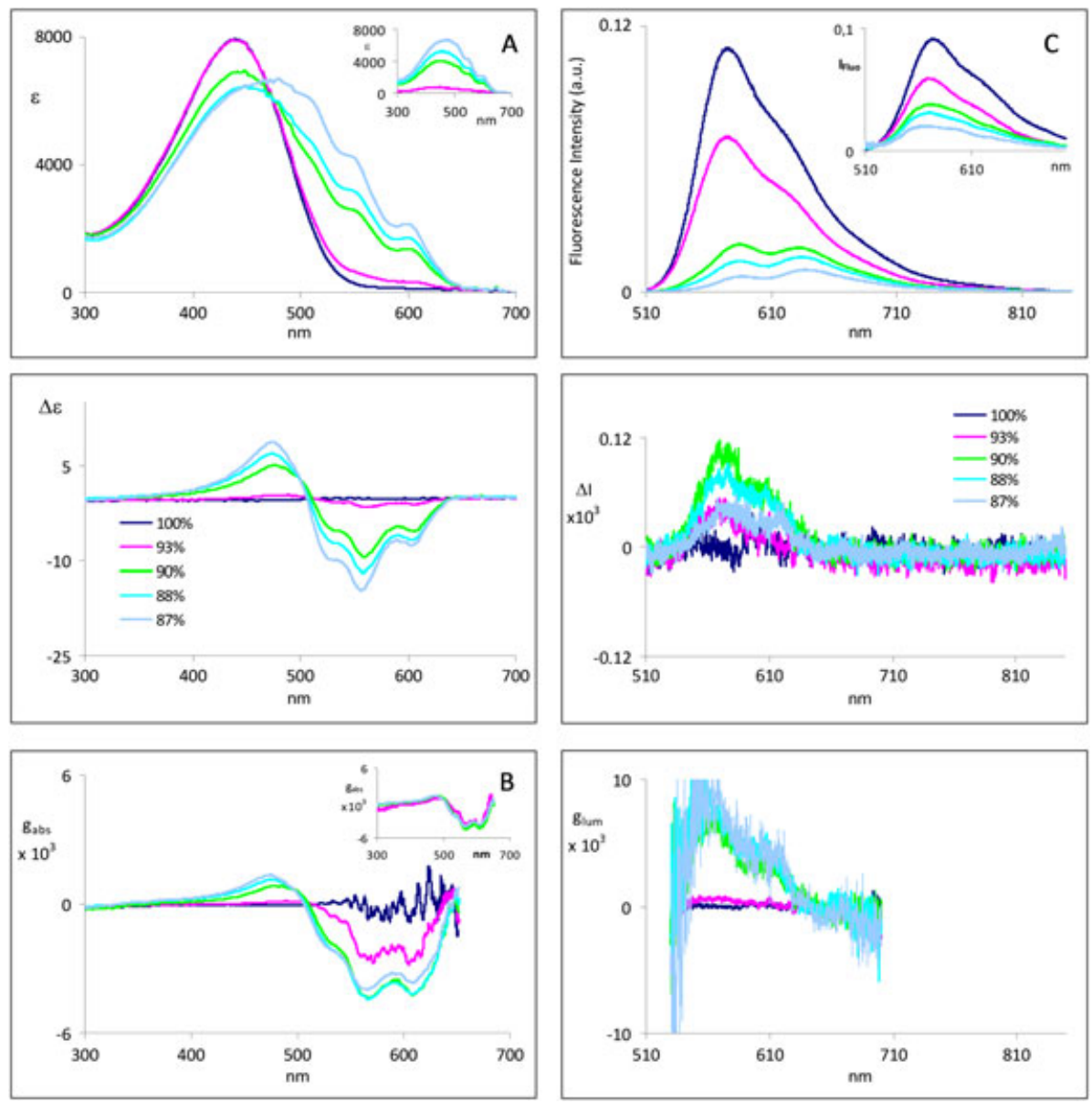

Fig. 3. (Left panels) Ultraviolet (top), electronic circular dichroism (middle), and $g_{\text {abs }}$ (bottom) spectra of poly[3-((S)-2-methylbutyl)-thiophene] (PMBT) in different $\mathrm{CHCl}_{3}$ /methanol mixtures. Inset A: ultraviolet spectra of the chiral component. Inset B: $g_{\mathrm{abs}}$ spectra of the chiral component.(Right panels) Emission (top), circularly polarized luminescence (CPL) (middle), and $g_{\text {lum }}$ (bottom) spectra of PMBT in different $\mathrm{CHCl}_{3} /$ methanol mixtures with a 493 -nm excitation. CPL and $g_{\text {lum }}$ are not corrected; for this reason, $g_{\text {lum }}$ cannot be compared with $g_{\text {abs. }}$. Inset C: corrected luminescence spectra, accounting for absorption of emitted light.
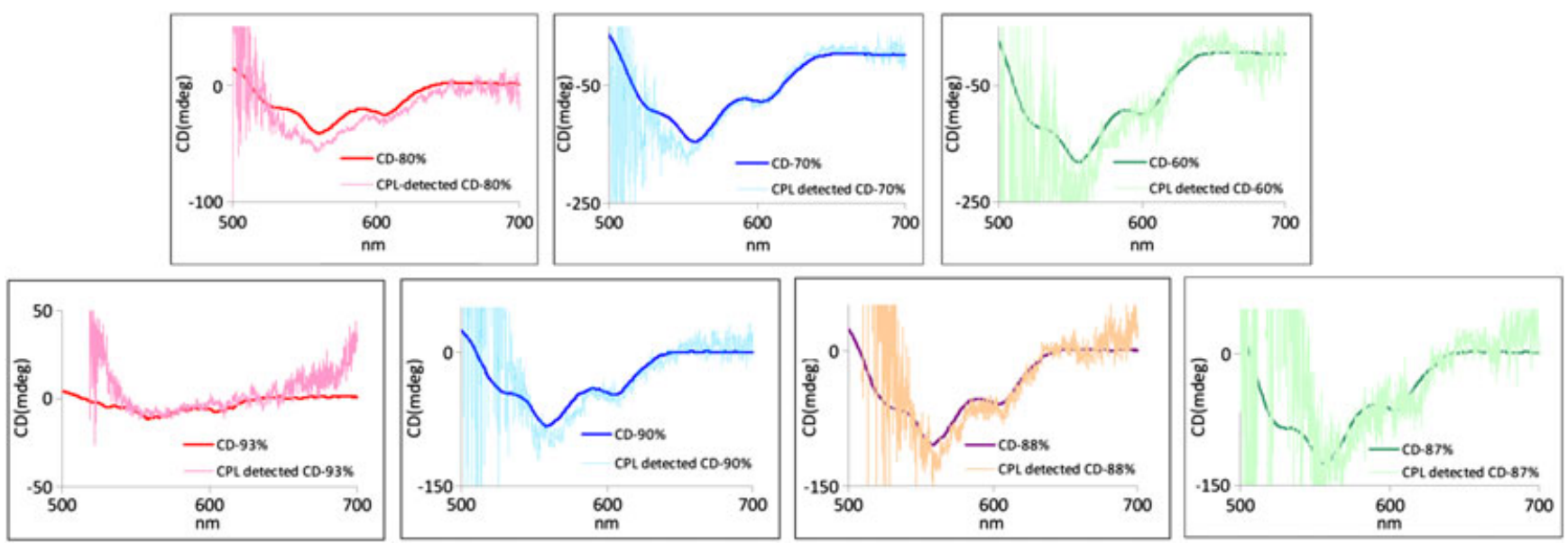

Fig. 4. (Top panels) Poly[3-((S)-2-methylbutyl)-thiophene] (PMBT) in $\mathrm{CHCl}_{3}$ /butanol mixtures. Comparison of circular dichroism (CD) spectra in mdeg measured on J815SE instrument (red, 80:20 (v/v); blue, 70:30 (v/v); dark green, 60:40 (v/v)) and of circularly polarized luminescence (CPL)-detected CD spectra calculated from the CPL and fluorescence signals obtained with a 470-nm excitation, as explained in the text (light pink, 80:20 (v/v); light blue, 70:30 (v/v); light green, 60:40 (v/v)). (Bottom panels) PMBT in $\mathrm{CHCl}_{3} /$ methanol mixtures. Comparison of CD spectra in mdeg measured on J815SE instrument (red, 93.7 (v/v); blue, 90:10 (v/v); brown, 88:12 (v/v); dark green, 87:13 (v/v)) and of CPL-detected CD spectra calculated from the CPL and fluorescence signals obtained with a 493-nm excitation, as explained in the text (light pink, 93:7 (v/v); light blue, 90:10 (v/v); light brown, 88:12 (v/v); light green, 87:13 (v/v)).

superposition brings us to the somewhat disappointing conclusion that what we have observed for PMBT is mainly $\mathrm{CD}$ rather than CPL. Of course, this possibility should be kept in mind whenever one deals with molecules exhibiting Chirality DOI: 10.1002/chir fluorescence in the same wavelength range, as $\mathrm{CD}$ and absorption; this indeed is not an infrequent instance. ${ }^{24}$

However, not all cases in Figure 4 are identical; some of them show a small difference in the two spectra. For this 


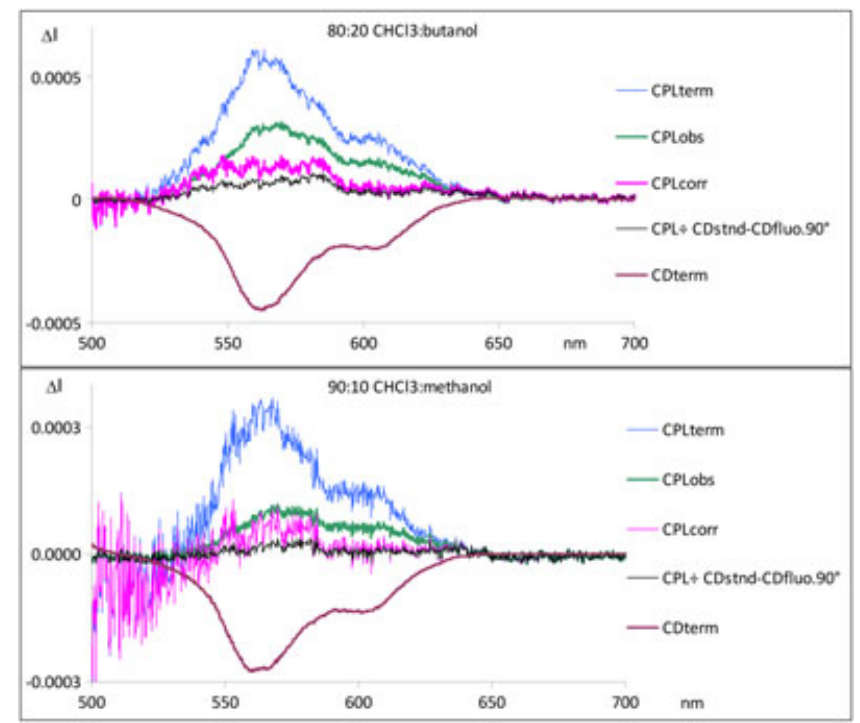

Fig. 5. Construction of $C P L_{\text {corr }}$ spectra of $\mathrm{PMBT}$ in the $80: 20 \mathrm{CHCl}_{3} /$ butanol mixed solution (top panel) and in the $90: 10 \mathrm{CHCl}_{3} /$ methanol mixed solution (bottom panel). The green traces are the observed circularly polarized luminescence $\left(\mathrm{CPL}_{\mathrm{obs}}\right)$ spectra; pink traces are corrected $\mathrm{CPL}$ spectra, $C P L_{\text {corr }}$, as obtained from eq. (9) of the Appendix section; they are to be compared with the black traces obtained from the difference of circular dichroism (CD) and CPL-detected CD spectra of Figure 4. The blue and brown traces are intermediate steps of the construction of the $C P L_{\text {corr }}$ spectra, as commented in the text and in the Appendix section.

reason, we strived to derive "true" $\mathrm{CPL}$ spectra. We first obtained them by subtracting the CPL-detected CD spectra from the corresponding $\mathrm{CD}$ one and opportunely multiplying them by the Transmittance spectrum. In the Appendix, we also devised a procedure for defining "true" or corrected CPL spectra, $C P L_{\text {corr }}$, from knowledge of the observed CPL spectra and of the different absorptions (or transmittances) of left and right circularly polarized light. The results for the two procedures are compared in Figure 5 for two cases, one presented in Figure 2 and one in Figure 3. The final results of the two procedures are the black curves and pink curves, respectively, the latter one being described by eq. (9) in the Appendix section. In Figure 5, we also report intermediate steps for the procedure of the Appendix: the green curve is the "observed" CPL spectra directly from the instrument, the blue curve is the CPL-term of eqs. (9) and (9') in the Appendix section, and the brown curve is the $\mathrm{CD}$ term of eqs. (9) and ( $\left.9^{\prime}\right)$ in the Appendix section. We think that the procedure derived in the Appendix section and illustrated in Figure 5 is applicable to all cases where Fluorescence and Absorbance phenomena in chiral molecules coexist, but it should be validated in a larger number of cases, especially when there are more evident differences between $\mathrm{CD}$ and CPL-derived CD spectra than observed here.

\section{CONCLUSIONS}

In the present paper, we have measured the UV, CD, fluorescence, and CPL spectra of solutions of PMBT (poly[3-((S)-2-methylbutyl)-thiophene]) in mixed solvents, consisting of two components, one of which is a good solvent (chloroform) and the other (either methanol or butanol) is a bad solvent for PMBT. The design of this work is not new, but the use of updated instrumentation has allowed to make the following steps forward:
1. a quantitative evaluation of the content of the chirally organized and of the nonchiral PMBT aggregates within the same solution;

2. because the UV spectra of the two types of aggregates are rather separate in wavelength, the CD spectra and the UV spectra of the chiral components are observed to increase with relative concentration of bad solvent in a parallel way, ensuring constant $g_{\text {abs }}$ spectra;

3. a similar statement about the behavior of $g_{\text {lum }}$ spectra cannot be made; indeed, the observed CPL signal is coming mostly from $\mathrm{CD}$, and the "true" CPL signal is quite small (see Figs. 4 and 5), the noise level being too high to draw a safe conclusion.

\section{APPENDIX}

In this section, we derive the "true" (i.e., corrected) Fluorescence and "true" CPL spectra from the observed Fluorescence and CPL spectra. We denote the latter data with the subscript "obs" and the former ones with the subscript "corr". We have

$$
\begin{aligned}
& C P L_{\mathrm{obs}}=I_{\mathrm{Lobs}}-I_{\mathrm{Robs}} \\
& 2 * F l u o_{\mathrm{obs}}=I_{\text {Lobs }}+I_{\text {Robs }}
\end{aligned}
$$

As explained in the text

$F l u o_{\text {corr }}=\frac{F l u o_{\text {obs }}}{T}$

( $T$ being the observed transmittance). Analogously, we may define

$C P L_{\text {corr }}=\frac{I_{\text {Lobs }}}{T_{\mathrm{L}}}-\frac{I_{\text {Robs }}}{T_{\mathrm{R}}}$

Combining eqs. (4) and (3), we obtain

$C P L_{\text {corr }}=\frac{1}{T^{2}}\left[F l u o_{\mathrm{obs}}\left(T_{\mathrm{R}}-T_{\mathrm{L}}\right)+\left(C P L_{\mathrm{obs}} / 2\right) *\left(T_{\mathrm{R}}+T_{\mathrm{L}}\right)\right]$

Of course, $T^{2}=T_{\mathrm{L}} T_{\mathrm{R}}$. To obtain the $T_{\mathrm{L}}$ and $T_{\mathrm{R}}$ data occurring in the CPL/fluorescence experiments from the absorbance and $\mathrm{CD}$ spectra recorded on a standard $\mathrm{CD} /$ absorbance spectrometer providing $\bar{A}$ and $\Delta \bar{A}$ data, respectively, we may start from the equation

$A=\bar{A} * \alpha=\log _{10}\left(\frac{1}{T}\right)$

where $A$ is the absorbance occurring in the CPL/fluorescence experiment and $\alpha$ keeps track of the different optical path of the $\mathrm{CD}$ and $\mathrm{CPL}$ experiment. It depends on the scattering geometry, on cuvette type and on path length: in the present case, $\alpha=2.5$. A similar relation exists between the $\mathrm{CD}$ spectrum experienced in the CPL experiments $\Delta A$ and the CD spectrum obtained in a standard spectrometer $\Delta A$, namely

$\Delta A=\overline{\Delta A} * \alpha=\log _{10}\left(\frac{T_{\mathrm{R}}}{T_{\mathrm{L}}}\right)$

Inverting eqs. (6) and (7) and opportunely combining them, we obtain

$T_{\mathrm{L}}=10^{-\alpha *(\bar{A}+\overline{\Delta A} / 2)}$ 
and

$T_{\mathrm{R}}=10^{-\alpha *(\bar{A}-\overline{\Delta A} / 2)}$

Substituting eqs. (8) and (8') into eq. (5) and keeping also in mind, from eq. (6), that $T=10^{-\alpha * \bar{A}}$, we have

$$
\begin{aligned}
C P L_{\mathrm{corr}}=\frac{1}{T} & {\left[\text { Fluo }_{\mathrm{obs}}\left(10^{\alpha * \overline{\Delta A} / 2}-10^{-\alpha * \overline{\Delta A} / 2}\right)\right.} \\
& \left.+\left(C P L_{\mathrm{obs}} / 2\right) *\left(10^{\alpha * \overline{\Delta A} / 2}+10^{-\alpha * \overline{\Delta A} / 2}\right)\right]
\end{aligned}
$$

Equation (9) is used in the text, and results are presented in Figure 5 as

$C P L_{\text {corr }}=C D_{\text {term }}+C P L_{\text {term }}$

A physically meaningful approximation of eq. (9) may be obtained by developing the exponentials in Taylor's series (indeed $\overline{\Delta A}$ is quite small) and keeping terms up to first order in $\overline{\Delta A}$. One, thus, obtains

$C P L_{\mathrm{corr}}=\frac{1}{T}\left[F l u o_{\mathrm{obs}}(\alpha * \ln (10) * \overline{\Delta A})+\left(C P L_{\mathrm{obs}}\right)\right]$

\section{ACKNOWLEDGMENTS}

We thank Italian "Ministero dell'Istruzione, dell'Università e della Ricerca" for the financial support, under the auspices of the program PRIN 2008LYSEBR. We thank Engineer Luca Berghella, University of Brescia, Italy, for helping us in the updating of the CPL apparatus.

\section{LITERATURE CITED}

1. Langeveld-Voss BMW, Janssen RAJ, Meijer EW. On the origin of optical activity in polythiophenes. J Mol Struct 2000;521:285-301.

2. Langeveld-Voss BMW, Janssen RAJ, Christianans MPT, Meskers SCJ, Dekkers HPJM, Meijer EW. J Am Chem Soc 1996;118:4908-4909.

3. Spano FC, Zhao Z, Meskers SCJ. Analysis of the vibronic fine structure in circularly polarized emission spectra from chiral molecular aggregates. J Chem Phys 2004;120:10594-10604.

4. Tempelaar R, Stradomska A, Knoester J, Spano FJ. Circularly polarized luminescence as a probe for long-range interactions in molecular aggregates. J Phys Chem B 2011;115:10592-10603.

5. Lebon F, Longhi G, Abbate S, Catellani M, Wang F, Polavarapu PL. Circular dichroism spectra of regioregular poly [3[(S)-2-methylbutyl]-thiophene] and Poly[3,4-di[(S)-2-methylbutyl]-thiophene]. Enantiomer 2002;7:207-212.

6. Lebon F, Longhi G, Abbate S, Catellani M, Luzzati S, Bolognesi A. Chiral polyalkylthiophenes for organic light emitting diodes. Mater Res Soc Symp Proc 2002;708:BB10.28.1-BB10.28.7.

7. Catellani M, Luzzati S, Bertini F, Bolognesi A, Lebon F, Longhi G, Abbate S, Famulari A, Meille SF. Chem Mater 2002;14:4819-4826.

8. Wang F, Polavarapu PL, Lebon F, Longhi G, Abbate S, Catellani M. Absolute configuration and conformational stability of (S)-(+)-3-(2-methylbutyl] thiophene and (+)-3,4-Di[(S)-2-methylbutyl]thiophene and their polymers. J Phys Chem A 2002;106:5918-5923.

9. Arosio P, Famulari A, Catellani M, Luzzati S, Torsi L, Meille SF. First detailed determination of the molecular conformation and the crystalline packing of a chiral poly(3-alkylthiophene): poly-3-(S)-2-methylbutylthiophene. Macromolecules 2007;40:3-5.

10. Castiglioni E, Abbate S, Longhi G. Revisiting with updated hardware an old spectroscopic technique: circularly polarized luminescence. Appl Spectrosc 2010;64:1416-1419.

11. Muller G, Maupin CL, Riehl JP, Birkedal H, Piguet C, Bünzli J-CG. Structural photophysical and chiro-optical properties of lanthanide complexes with bis(benzimidazole)pyridene-based chiral ligand. Eur J Inorg Chem 2003;4065-4072.

12. Field JE, Muller G, Riehl JP, Venkataraman D. Circularly polarized luminescence from bridged triarylamine helicenes. J Am Chem Soc 2003;125:11808-11809.

13. Harada T, Nakano Y, Fujiki M, Naito M, Kawai T, Hasegawa Y. Circularly polarized luminescence of $\mathrm{Eu}$ (III) complexes with point- and axis-chiral ligands dependent on coordination structures. Inorg Chem 2009;48: 11242-11250.

14. Nakano Y, Liu Y, Fujiki M. Circularly polarized luminescence from poly (9,9-di- $n$-decylfluorene) by terpene chirality transfer. Polym Chem 2010;1:460-469.

15. Lakhwani G, Koeckelberghs G, Meskers SCJ, Janssen RAJ. The chiroptical properties of chiral substituted poly3-((3S)-3,7-dimethyloctyl) thiophene] as a function of film thickness. Chem Phys Lett 2007; 437:193-197.

16. Berghella L. Progettazione e realizzazione di un sistema di controllo, scansione e acquisizione dati installato su uno spettrometro per misure di dicroismo circolare. Undergraduate Thesis, Engineering and Information School, University of Brescia, Italy, 2009.

17. Dekkers HPJM, Circularly polarized luminescence: a probe for chirality in the excited state. In: Berova N, Nakanishi K, Woody RA, editors. Circular Dichroism: Principles and Applications, 2nd ed. New York: J. Wiley \& Sons, Inc.; 2000, p 185-215. ISBN 0-471-33003-5.

18. Blok PML, Dekkers HPJM. Measurement of the circular polarization of the luminescence of photoselected samples under artifact-free conditions. Appl Spectrosc 1990;44:305-309.

19. Shindo Y, Nakagawa M. On the artifacts in circularly polarized emission spectroscopy. Appl Spectrosc 1985;39:32-38.

20. Dekkers HPJM, Closs LE. The optical activity of low-symmetry ketones in absorption and emission. J Am Chem Soc 1976;98:2210-2219.

21. Castiglioni E, Longhi G, Lebon F, Abbate S. Updating circularly polarized luminescence (CPL) experimentation with some changes in the hardware and design. Poster \#1, CD2011, The $13^{\text {th }}$ International Conference on Chiroptical Spectroscopy, Oxford UK 24-28 July 2011-10-24.

22. Brittain HG, Richardson FS. Circularly polarized emission studies on the chiral nuclear magnetic resonance lanthanide shift reagent tris (3-trifluoroacetyl- $d$-camphorato)europium(III). J Am Chem Soc 1976; 98:5858-5863.

23. Castiglioni E, Abbate S, Longhi G, Gangemi R, Lauceri R, Purrello R. Absorption flattening as one cause of distortion of circular dichroism spectra of $-\mathrm{RuPhen}_{3} \cdot \mathrm{H}_{2}$ TPPS complex. Chirality 2007;19:642-646.

24. Kawagoe Y, Fujiki M, Nakano Y. Limonene magic: noncovalent molecular chirality transfer leading to ambidextrous circularly polarised luminescent $\pi$ conjugated polymers. New J Chem 2010;34:637-647. 